\title{
Electrocardiogram Profile in Children with Dengue Infection at Dr. Hasan Sadikin General Hospital and Bandung City Hospital
}

\author{
Sakinah Binti Shabbir Hussain, ${ }^{1}$ Rahmat Budi Kuswiyanto, ${ }^{2}$ Januarsih Iwan, ${ }^{3}$ \\ ${ }^{1}$ Faculty of Medicine Universitas Padjadjaran, ${ }^{2}$ Department of Child Health Faculty of Medicine, \\ Universitas Padjadjaran/Dr. Hasan Sadikin General Hospital Bandung, ${ }^{3}$ Departement of Anatomy \\ and Cell Biology, Faculty of Medicine, Universitas Padjadjaran
}

\begin{abstract}
Background: Dengue is a mosquito-borne infection which causes many manifestations on the organs, mainly heart. According to the histopathological analysis in heart, there is a direct action of dengue virus towards myocardium. This study was an initial study of cardiac involvement in dengue infection using electrocardiogram as a measurement tool.

Methods: This study was a descriptive cross-sectional study and data collection were performed consecutively. A total of 17 children was taken which had been diagnosed with dengue infection according to World Health Organization criteria and had fulfilled inclusion and exclusion criteria of this study in Dr. Hasan Sadikin General Hospital and Bandung City Local Hospital. Once informed consent was obtained, the electrocardiogram procedure was performed according to standard electrocardiogram procedure in pediatrics. Data were analyzed by a pediatric cardiology consultant. Data were interpreted based on the electrocardiogram characteristics according to the type of dengue infection.

Results: P wave amplitude was getting shorter as the severity of dengue infection increased. P wave duration increased slightly with increased severity of dengue infection. There was a significant increase in PR interval as the severity of dengue infection increased. The same was also true for ST and QTc wave.

Conclusions: The pattern of electrocardiogram profile in children with dengue infection were within normal range. [AMJ.2016;3(4):629-32]
\end{abstract}

Keywords: Cardiac Involvement, Dengue Infection, Electrocardiogram

\section{Introduction}

One of the most important mosquito-borne viral diseases in world is dengue infection. ${ }^{1}$ Dengue is a member of flaviviridae family consisting of a single stranded RNA virus with four serotypes: DENV-1, DENV-2, DENV-3 and DENV4. ${ }^{2}$ The transmission was facilitated by a specific mosquito called Aedes Aegypti. ${ }^{3}$ Dengue virus has been reported to infect many organs in human body, mainly heart. ${ }^{4}$ Few cardiac complication has been reported including atrial fibrillation, ${ }^{5}$ atrioventricular block, $^{6}$ and myocarditis. ${ }^{7}$ According to the histopathological analysis, dengue virus has a direct action on myocardium ${ }^{8}$ through autoimmune response against cardiac epitopes. $^{9}$ Therefore, to detect myocarditis, electrocardiogram widely is used as a screening tool, not only because it is easily available but also due to its ability to indicate the extend of acute myocardial injuries. ${ }^{10,11}$

Several studies had been carried out earlier, including the one in Columbia ${ }^{12}$ where out of 102 dengue hemorrhagic fever patients, 11 of them diagnosed as suffering from myocarditis, which was confirmed by electrocardiogram and echocardiograph analysis, and another study in Sri Lanka ${ }^{13}$ where an elevation of cardiac enzyme in patients with dengue infection had been proven to include cardiac involvement in dengue infection patients. Hence, an initial study of cardiac involvement due to dengue infection using electrocardiogram as a tool of measurement is necessary. The objective was to know the electrocardiogram profile in patients with dengue fever, dengue hemorrhagic fever, and dengue shock syndrome. This type of study has never been carried out before in this hospital.

Correspondence: Sakinah Binti Shabbir Hussain, Faculty of Medicine, Universitas Padjadjaran, Jalan Raya BandungSumedang Km.21, Jatinangor, Sumedang, Indonesia, Phone: +6287822004447 Email: sweetycramel1991@yahoo.com 


\section{Methods}

This was a descriptive cross sectional study. Data collected in this study were primary data collected through standard electrocardiogram procedure in children suffering from dengue infection. This study was carried out during early September until early November 2014 in The pediatric ward in Dr. Hasan Sadikin Hospital and Bandung City Local Hospital after approval was gained from the Ethic Committee of the respective hospitals.

Children with dengue infection who met inclusion and exclusion criteria were sampled consecutively. The inclusion criteria are all children who had been diagnosed with dengue virus infection at the pediatric department whose age was 14 years old or younger in both hospitals. Parents and guardians of these children were given written and verbal informed consent and they had agreed to the electrocardiogram procedure. The exclusion criteria consisted of children detected having any congenital heart disease, abnormal genetic condition, disease around patient's chest area, and also other health conditions like pneumothorax and sepsis.

Children with dengue infection was diagnosed according to the World Health Organization ( WHO ) guidelines. ${ }^{14}$ The ECG-12 monitoring was used with 12 leads attached to the subject according to the manufacturer's instruction. Six precordial leads and six extremities leads are used.15 Then the electrocardiogram data were recorded: pulse rate, respiratory rate, and heart rate. Data on the temperature were also, and $t$ obtained during electrocardiogram procedure by placing a thermometer under the axilla. Initial haemoglobin, hematocrit, and thrombocyte counts were also recorded.

Once data have been recorded, the electrocardiogram data were analyzed by a pediatric cardiologist consultant. Data from the electrocardiogram profile were tabulated according to the type of infection, including dengue fever, dengue hemorrhagic fever, and dengue shock syndrome. The characteristics of electrocardiogram were recorded according to rhythm, $\mathrm{P}$ wave amplitude, $\mathrm{P}$ wave duration, QRS complex amplitude, QRS complex axis, QRS complex duration, ST segment, T wave, and QTc interval respectively, according to the type of infection. Data from the interpreted electrocardiogram of the children according to the type of infections were analyzed

\section{Results}

During the study period, a total of 29 children that were admitted and diagnosed as suffering from dengue infection in both hospitals. However, out of 29 only 17 children met the requirements of the inclusion criteria. From these 17 children, the proportion of boys to girls was 1 to 1 . Out of 17 children, 7 children

Table 1 Characteristics of Vital Signs and Laboratory Examination

\begin{tabular}{lccc}
\hline \multicolumn{1}{c}{ Mean/Median } & $\begin{array}{c}\text { Dengue fever } \\
\mathbf{n = 7}\end{array}$ & $\begin{array}{c}\text { Dengue hemorrhagic } \\
\text { fever } \\
\mathbf{n = 6}\end{array}$ & $\begin{array}{c}\text { Dengue shock } \\
\text { syndrome } \\
\mathbf{n = 4}\end{array}$ \\
\hline Age (years) & $5.6 \pm 2.4$ & $5.67 \pm 4.0$ & $8.5(7-12)$ \\
Weight (kilograms) & $17.0(12.0-68.5$ & $14.0(11.0-31.0)$ & $31.5(20-36)$ \\
Temperature ( $\left.{ }^{\circ} \mathrm{c}\right)$ & $36.8 \pm 0.4$ & $35.6 \pm 0.9$ & $36.9(36.5-37.4)$ \\
Respiratory rate (times per minute) & $25.9 \pm 2.3$ & $27.0(26-30)$ & $25.5(23-30)$ \\
Pulse rate (times per minute) & $100.9 \pm 4.5$ & $108.3 \pm 9.2$ & $99.0(72-128)$ \\
Heart rate (Bpm) & $103.9 \pm 19.4$ & $100.5 \pm 23.5$ & $91.0(76-97)$ \\
Blood pressure (mmHg) & & & $90.0(80-108)$ \\
$\quad$ Systole & $90.0(90-100)$ & $89.2 \pm 9.2$ & $55.0(50-90)$ \\
$\quad$ Diastole & $60.0(60-70)$ & $60.0(50-65)$ & $14.1(11-18)$ \\
Haemoglobin(g/dL) & $14.3 \pm 3.1$ & $13.7 \pm 2.4$ & $41.3(31-51)$ \\
Hemotocrit(\%) & $36.2 \pm 4.0$ & $39.8 \pm 6.1$ & $23.0(13-83)$ \\
Thrombocyte(uL) & $89.6 \pm 51.4$ & $58.3 \pm 30.1$ &
\end{tabular}


Table 2 Electrocardiogram profile according to WHO dengue infection classification

\begin{tabular}{lcccc}
\hline \multicolumn{1}{c}{ Mean / Median } & $\begin{array}{c}\text { Dengue Fever } \\
\mathbf{n = 7}\end{array}$ & $\begin{array}{c}\text { Dengue } \\
\text { Hemorrhagic } \\
\text { Fever } \\
\mathbf{n = 6}\end{array}$ & $\begin{array}{c}\text { Dengue Shock } \\
\text { Syndrome } \\
\mathbf{n = 4}\end{array}$ \\
\hline P wave & Amplitude (mV) & $0.12(0.08-0.30)$ & $0.09(0.05-0.10)$ & $0.08(0.05-0.10)$ \\
PR Interval (ms) & Duration (ms) & $80.3(80-82)$ & $81.0 \pm 12.9$ & $82.0(80-88)$ \\
QRS & Amplitude(mV) & $129.7 \pm 17.4$ & $151.7 \pm 50.1$ & $179.0(114-224)$ \\
complex & Axis( $\left(^{\circ}\right)$ & $63.9 \pm 32.0$ & $1.7 \pm 1.0$ & $1.6(1.1-3.2)$ \\
ST Segment (ms) & Duration (ms) & $80.0 \pm 3.9$ & $74.0 \pm 3(68-103)$ & $77.0(66-82)$ \\
T wave (ms) & & $80.0(80-125)$ & $140.0 \pm 56.2$ & $140.0(120-160)$ \\
QTc interval (ms) & & $160.0(120-160)$ & $146.7 \pm 41.3$ & $150.0 \pm 50.3$ \\
\hline
\end{tabular}

Note: $\mathrm{X} \pm \mathrm{SD}=\mathrm{Mean} \pm$ Standard Deviation, $\mathrm{y}($ Min-Max) $=$ Median( Minimum Value-MaximumValue), $\mathrm{mV}=$ Millivolt, $\mathrm{ms}=$ Millisecond, ${ }^{\circ}=$ Degree

were diagnosed as suffering from dengue fever, 6 children were diagnosed as having dengue hemorrhagic fever, and 4 children experienced dengue shock syndrome. Prior to electrocardiogram procedure was, vital signs and laboratory examination results had been assessed.

As shown in Table 1, age and weight were recorded from the medical status. Overall vital signs, such as temperature, vital sign, pulse pressure, heart rate, and blood pressure were shown to be in the normal range. The laboratory data included initial haemoglobin,hematocrit, and haemoglobin counts.

From the data in the table, the $\mathrm{P}$ wave amplitude became shorter as the severity of the dengue infection increased, i.e. from a mean of 0.12 milivolt in dengue to 0.09 milivolt in dengue hemorrhagic fever and much horter shorter in dengue shock syndrome. P wave duration increased slightly from dengue fever to dengue shock syndrome. However, the PR interval duration, duration increased as the severity of the infection increased. The duration of ST segment increased as the severity of infection increased, i.e. from 80.00 milisecond in dengue fever to 140.00 milisecond in dengue hemorrhagic fever and dengue shock syndrome. Lastly, for QTc, the duration became shorter as severity of dengue infection increased.

\section{Discussion}

The electrocardiogram profile patterns in children with dengue infection in this study were within normal range. However there were certain changes in the value of electrocardiogram profile as the severity of dengue infection increased, which might show early signs of cardiac involvement.

The P wave duration increased as severity dengue infection increased, indicating the probability of atrial enlargement. Atria enlargement causes a delay in depolarization from sinoatrial node. The increment of PR intervalmightshow an earlysign of some cardiac problems due to a delay in conduction impulse from atrioventricular node, bundle of his, or bundle branch. The ST segment encompassed the end of ventricular depolarization to the beginning of ventricular repolarization. ${ }^{15}$ This increased in dengue hemorrhagic fever and dengue shock syndrome, compared to dengue fever. Although the range of increase was still in the normal range, there was a possibility that this showed early signs of acute myocarditis as shown in many studies that suggested dengue viral infection causes abnormalities in ST segment and a case report that had proven this assumption. ${ }^{16}$

Moreover, the proportion of boys to girls was approximately in this study, which supports a study carried out in Southern Vietnam ${ }^{17}$ showing that sex does not play much role in getting dengue infection.

Although the outcomes from this study show changes in electrocardiogram profile as the severity of infection increases, all values are still in the normal range and there is no evidence of cardiac involvement as shown in the previous study in Colombo. ${ }^{12}$ This condition might be due to a small number of 
dengue cases in this study which emphasizes the needs for a study with large sample size to reflect the population clearly.

Despite insignificant findings in this study on cardiac involvement in dengue infection, the pattern of the electrocardiogram profile indicates that there is a possibility of cardiac involvement. Therefore, a future study with more subjects and other measurement tools to show the cardiac involvement in dengue infection is necessary and may utilize this study as a starting point

\section{References}

1. Guzman MG, Halstead SB, Artsob H, Buchy P, Farrar J, Gubler DJ, et al. Dengue: a continuing global threat. Nat Rev Microbiol 2010;8:S7-16.

2. Simmons CP, Farrar JJ, van Vinh Chau $\mathrm{N}$, Wills B. Dengue. N Eng J Med. 2012;366(15):1423-32.

3. Salazar MI, Richardson JH, Sánchez-Vargas I, Olson KE, Beaty BJ. Dengue virus type 2: replication and tropisms in orally infected Aedes aegypti mosquitoes. BMC Microbiol. 2007;7(1):9.

4. Satarasinghe RL, Arultnithy K, Amerasena NL, Bulugahapitiya U, Sahayam DV. Asymptomatic myocardial involvement in acute dengue virus infection in a cohort of adult Sri Lankans admitted to a tertiary referral centre. Br J Cardiol 2007;14(3):171-3.

5. Veloso HH, Ferreira Júnior JA, Paiva JMBd, Honório JF, Bellei NCJ, Paola AAVd. Acute atrial fibrillation during dengue hemorrhagic fever. Braz J Infect Dis. 2003;7(6):418-22.

6. Sharma JK, Zaheer S. Variable atrioventricular block in dengue fever. I Indian Acad Clin Med. 2014;15(3-4):252-4.

7. Lee I-K, Lee $\mathrm{W}-\mathrm{H}$, Liu J-W, Yang KD. Acute myocarditis in dengue hemorrhagic fever: a case report and review of cardiac complications in dengue-affected patients. Int J Infect Dis 2010;14(10):e919-22.
8. Miranda $\mathrm{CH}$, de Carvalho Borges $\mathrm{M}$, Schmidt A, Pazin-Filho A, Rossi MA, Ramos SG, et al. A case presentation of a fatal dengue myocarditis showing evidence for dengue virus-induced lesion. Eur Heart J Acute Cardiovasc Care. 2013:2(2):127-30.

9. Shauer A, Gotsman I, Keren A, Zwas DR, Hellman Y, Durst R, et al. Acute viral myocarditis: current concepts in diagnosis and treatment. Isr Med Assoc J. 2013;15(3):180-5.

10. Fairweather D, Abston ED, Coronado MJ. Biomarkers of heart failure in myocarditis and dilated cardiomyopathy. In: Cihakova D, editor. Myocarditis. Rijeka, Croatia: InTech Open Access Publisher; 2011. p. 323-48.

11. Ukena C, Mahfoud F, Kindermann I, Kandolf R, Kindermann M, Böhm M. Prognostic electrocardiographic parameters in patients with suspected myocarditis. Eur J Heart Fail. 2011;13(4):398-405.

12. Salgado DM, Eltit JM, Mansfield K, Panqueba C, Castro D, Vega MR, et al. Heart and skeletal muscle are targets of dengue virus infection. Pediatr Infect Dis J. 2010;29(3):238-42.

13. Wichmann D, Kularatne S, Ehrhardt S, Wijesinghe S, Brattig NW, Abel W, et al. Cardiac involvement in dengue virus infections during the 2004/2005 dengue fever season in Sri Lanka. Southeast Asian J Trop Med Public Health 2009;40(4):72730.

14. Ranjit S, Kissoon N. Dengue hemorrhagic fever and shock syndromes. Pediatr Crit Care Med 2011;12(1):90-100.

15. Thaler MS. The only EKG book you'll ever need. Philadelphia: Lippincott Williams \&Wilkins; 2010.

16. Rajapakse S. Dengue shock. J Emerg Trauma Shock. 2011;4(1):120-7.

17. Thai KT, Binh TQ Giao PT, Phuong HL, Hung LQ Nam NV, et al. Seroprevalence of dengue antibodies, annual incidence and risk factors among children in southern Vietnam. Trop Med Int Health. 2005;10(4):379-86. 